\title{
Positive outcome of occlusal freeway space reestablishment in patients with medication overuse due to chronic migraine
}

\author{
H. Didier • C. Marchetti - A. Marchetti • \\ D. D'Amico $\cdot$ V. Tullo $\cdot$ A. Proietti Cecchini • \\ P. Di Fiore $\cdot$ G. Bussone $\cdot$ F. Santoro
}

(C) Springer-Verlag Italia 2013

\begin{abstract}
Considering the great chapter of migraines, it is important to note the signs and symptoms caused by an alteration of the relationship of the facial musculature and the occlusal freeway space (FWS) that is the distance from maximal intercuspation to the habitual rest position (measurable in 1.4-2.5 mm). To any mandible position changing (detected by periodontal, muscle and joint proprioceptors), there is an influence on the neuromuscular system and then an alteration of the FWS. A group of 60 patients with chronic migraine (CM) underwent a withdrawal of overused medication and were subjected to electromyographic and kinesiographic evaluation. All those who presented an alteration of the FWS at rest position have been subjected to treatment with orthosis device for about 12 months. The aim of our work is to obtain and define a correct mandibular position, physiological, in agreement with the neuromuscular structures of the patient. Clinical results obtained on this pool of patients, in terms of reduction of the VAS, let us understand the importance of the inviolability of the FWS and to eliminate any type of mandibular deviation with respect to the closure trajectory induced by TENS in patients suffering from CM.
\end{abstract}

\footnotetext{
H. Didier · C. Marchetti - A. Marchetti ( $)$ · F. Santoro Dipartimento Scienze Chirurgiche Ricostruttive e Diagnostiche, Fondazione IRCCS Cà Granda, Ospedale Maggiore Policlinico, Clinica Odontoiatrica, Università degli Studi di Milano,

Via della Commenda, 10, 21022 Milan, Italy

e-mail: andrea.marchetti15@gmail.com

D. D’Amico - V. Tullo · A. Proietti Cecchini - P. Di Fiore ·

G. Bussone

Headache Unit, Department of Clinical Neuroscience,

C. Besta Neurological Institute and Foundation, Milan, Italy
}

Keywords Medication overuse (MO) $\cdot$ Chronic migraine (CM) - Gnathological evaluation · Orthosis · TENS ·

Kinesiograph

\section{Introduction}

Temporomandibular disorders (TMD) may have a role in headache patients; TMD can be one of the possible causes of headache or facial pain, but they also should coexist with primary headaches. Recently, it has been suggested that TMD may have a specific role in the progression from episodic migraine into chronic migraine (CM) $[1,2]$.

$\mathrm{CM}$ patients are often difficult to treat, particularly when medication overuse (MO) is associated. An area of particular interest in these patients is represented by the signs and symptoms caused by an alteration of the mandibular posture and to the respect of the occlusal freeway space (FWS).

The freeway space is the distance from maximal intercuspation to the habitual rest position; in this position we spend most of our life and it is the position in which all mandibular movements start and return (speaking, swallowing and chewing) [3].

The FWS must be assessed in the three planes of the space. The occlusal free way space is altered by any variation of the jaw position [4].

The correction of deviations recorded in the three planes of the space is achieved and maintained by the orthosis device, simulator of the new spatial position of the mandible in contrast to the common bite used as deprogrammers [5].

The objective of the study is to evaluate the correction's effects of the cranio-mandibular relationship in patients with chronic migraine. 


\section{Materials and methods}

A sample of 60 consecutive patients with MO due to CM (13 men and 47 women), admitted as inpatients at the Headache Unit of the Neurological Institute Carlo Besta to undergo a withdrawal protocol to stop MO, was enrolled. All of them was affected by MO [6].

Gnathological clinical and instrumental evaluation included: pain evaluation by VAS, seeking parafunctional habits (bruxism and clenching) for TMJ noises and muscle pain on palpation of the masseter, temporal and sternocleidomastoid, kinesio-electromyographic examination, described elsewhere, [7] before and after stimulation with TENS applied to the jaw's coronoid incisure to locate the occlusal freeway space and then the correct mandibular position in agreement with neuromandibular structures [8]. The TENS used had a pulse duration of $500 \mu$ s, intensity from 0 to $25 \mathrm{~mA}$ and a frequency of 40 pulses $/ \mathrm{min}$.

Instrument used was computer mandibular scanning K7 Myo-Tronic Seattle.

Patients with a lateral deviation higher than $0.4 \mathrm{~mm}$ compared to the mandibular rest position induced by TENS were considered as candidates for orthosis, according to a previous report from our group [6].

\section{Results}

All 60 patients investigated had one or more signs of TMJ Internal Derangement: noises $55 \%$, pain $73 \%$, limited mouth opening of $45 \% .76 \%(n=46)$ showed parafunctions: $11(24 \%)$ clenching, $8(17 \%)$ grinding and 27 (59\%) both. $44(73 \%)$ showed pain on palpation of the temporal muscle, masseter and sternocleidomastoid.

All 60 patients were subjected to Kinesio-EMG examination, and of these, $93 \%(n=56)$ showed an alteration of the freeway space at habitual mandibular rest position in defect before the application of TENS (mean $1.3 \mathrm{~mm}$ ). After application of TENS (at physiological rest position), all of these patients $(n=56)$ showed an increase of occlusal freeway space (mean $3.2 \mathrm{~mm}$ ). The average increase of the freeway space was around at $1.9 \mathrm{~mm}$.

An orthosis device was applied on the 44 patients with mandibular lateral deviation $\geq 0.4 \mathrm{~mm}$; the re-assessed patients at follow-up at 6 months, after treatment with orthosis device, showed a reduction in the headache intensity: the mean VAS was reduced from 5.2 (SD 1.8) to 3.3 (SD 1.8).

We have seen two distinct issues in our pool of patients: joint problems (internal derangement) and myofascial pains.

- Patients with joint issues present symptoms, such as articular noises $(n=33-55 \%)$ and/or articular pain $(n=44-73 \%)$ and/or restriction on mouth's opening $(n=27-45 \%)$.

- Patients with myo-fascial problems present pain at manual compression of masticatory muscles: left masseter muscle (LM), $n=44-73 \%$; right masseter (RM), $n=41-68 \%$; left temporal muscle (LT), $n=38-63 \%$; right temporal (RT), $n=36-60 \%$; left sternocleidomastoid (LSCM), $n=34-57 \%$; right sternocleidomastoid (RSCM), $n=29-48 \%$ (Fig. 1).

\section{Discussion and conclusion}

Although not always recognized, the alteration of craniomandibular relationship and postural balance of the facial musculature, expressed by the respect of the occlusal FWS, are potential comorbidity of migraine and possible factors of its chronicity.

The violation, in excess as in defect, of the FWS at mandibular rest position, and the mandibular closing
Fig. 1 Left masseter muscle $(L M)$, right masseter $(R M)$, left temporal muscle $(L T)$, right temporal $(R T)$, left sternocleidomastoid (LSCM), right sternocleidomastoid (RSCM)

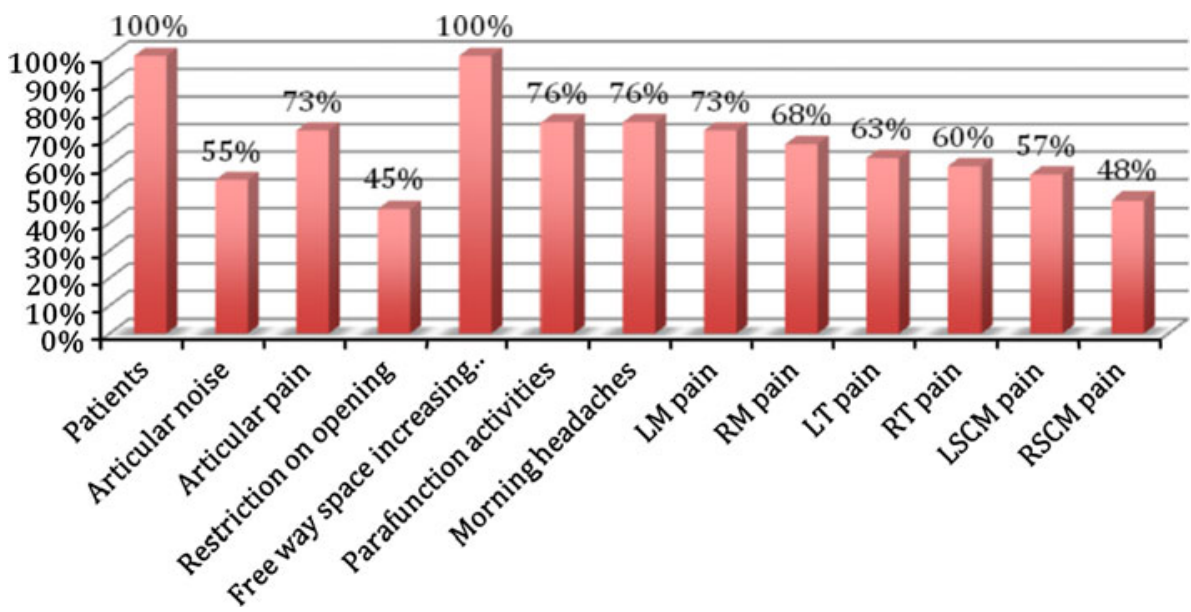


trajectory, can lead to undesirable responses of the craniomandibular joint's components and neuromuscular equilibrium of the patient [9].

Altered muscular activity, such as contracture sustained over time, and muscle spasm, associated with shortening of the muscle fibers, may help explain the phenomena that underlie the onset of trigger points. These points are presented with painful compression and can evoke characteristically referred pain even in relatively remote locations and sometimes impressive autonomic phenomena [10].

For these reasons, any spatial variation of the mandibular structures toward the skull base is signaled through a complex system of proprioception, conscious and unconscious, able to influence the phasic and tonic activity of the neuromuscular system.

Dental malocclusion, understood as a dysfunction related to the jaw lowering and elevator muscles, would not to be the only cause of abnormal contractile activity of the muscular system and it is clear that the restoration of a normal physiological tone of the muscles, related to mandibular spatial position, is available through the achievement of an harmonious muscular balance due to a proper postural mandibular-cranial-spinal relationship [11]. Every muscle has an unalterable resting length that tends to rediscover each time it is changed [12].

The results of this study suggest that these alterations may have a high prevalence in patients with $\mathrm{CM}$, but especially that patients treated with the orthosis device can report a reduction of the intensity of headache corresponding to the decrease of the values of the VAS.

The obtained data show how the length of the muscle fibers would be changed recovering the physiological mandibular position by re-establishing the correct craniomandibular relationship and eliminating three-dimensional mandibular deviations during mandibular closure trajectory. This therapeutic intervention can reduce pain in patients with chronic headaches associated with MO, probably through the reduction of nociceptive inputs from masticatory muscle, a mechanism which in fact may be relevant in the complex pathophysiology of peripheral and central sensitization at the basis of migraine progression into $\mathrm{CM}$ and $\mathrm{MO}[1]$.

Conflict of interest The authors certify that there is no actual or potential conflict of interest in relation to this article.

\section{References}

1. Bevilaqua Grossi D, Lipton RB, Bigal ME (2009) Temporomandibular disorders and migraine chronification. Curr Pain Headache Rep 13(4):314-318

2. Gonçalves DA, Camparis CM, Speciali JG, Franco AL, Castanharo SM, Bigal ME (2011) Temporomandibular disorders are differentially associated with headache diagnoses: a controlled study. Clin J Pain 27(7):611-615

3. Konchak PA, Thomas NR, Lanigan DT, Devon RM (1988) Freeway space measurement using mandibular kinesiograph and EMG before and after TENS. Angle Orthod 58(4):343-350

4. Trulsson M (1993) Multiple-tooth receptive fields of single human periodontal mechanoreceptive afferents. J Neurophysiol 69(2):474-481

5. Jankelson R (1997) Effect of vertical and horizontal variants on the resting activity of masticatory muscles. Anthol Craniomandib Orthop IV:69-76

6. Committee Headache Classification, Olesen J, Bousser MG, Diener HC, Dodick D, First M, Goadsby PJ, Göbel H, Lainez MJ, Lance JW, Lipton RB, Nappi G, Sakai F, Schoenen J, Silberstein SD, Steiner TJ (2006) New appendix criteria open for a broader concept of chronic migraine. Cephalalgia 26(6):742-746

7. Didier H, Marchetti C, Marchetti A, D'Amico D, Tullo V, Bussone G, Santoro F (2012) Implementing gnathological and neuromuscular concepts in patients with chronic migraine. Neurol Sci 33(1):S177-S180

8. Franco M, Ferronato G, Peretta R, Borgo G (1993) La recidiva nella chirurgia ortognatica delle terze classi. Atti VIII Congresso Nazionale della Società di Chirurgia Maxillo-Facciale 535-537

9. Lavelle ED, Lavelle W, Smith HS (2007) Myofascial trigger points. Anesthesiol Clin 25(4):841-851

10. Cooper BC, Kleinberg I (2008) Establishment of a temporomandibular physiological state with neuromuscular orthosis treatment affects reduction of TMD symptoms in 313 patients. J Craniomandib Pract 26(2):104-117

11. Lippold C, Danesh G, Hoppe G, Drerup B, Hackenberg L (2006) Sagittal spinal posture in relation to craniofacial morphology. Angle Orthod 76(4):625-631

12. George JP, Boone MEJ (1979) A clinical study of rest position using the Kinesiograph and Myomonitor. Prosthet Dent 41(4): $456-462$ 